\title{
Efectos de una estrategia de intervención educativa para la incorporación de la actividad física como hábito en jóvenes de la Universidad Surcolombiana de la asignatura 'deporte formativo', semestre 2013-2
}

\author{
Mario German Rodríguez Mondragón \\ Universidad Surcolombiana, Colombia \\ mariogermanrodriguez@gmail.com \\ Adolfo Martínez Castro \\ Universidad de Pamplona, Colombia
}

\section{Resumen}

La investigación denominada “Efectos de una estrategia de intervención educativa en el desarrollo de hábitos y estilos de vida saludable en jóvenes de la Universidad Surcolombiana de la asignatura deporte formativo semestre B 2013." Es un trabajo realizado con estudiantes de la cátedra de deporte formativo de la Universidad Surcolombiana, para fomentar la actividad física como hábito para que pueda ser asimilado dentro del estilo de vida de los jóvenes universitarios, se utilizaron algunos parámetros de la condición física para que percibieran cambios favorables y de esta manera generarles gratificación, la intervención tambien utilizó una metodología con un alto componente lúdico con el fín de hacer más atractiva la clase. Los resultados evidencian las bondades de la intervención al comprobarse cambios estadisticamente significativos en muchas de las variables estudiadas.

Palabras clave: estilo de vida saludable, deporte formativo, jóvenes universitarios, conductas patógenas, conductas inmunógenas.

Effects of an educational intervention strategy for the incorporation of physical activity as a habit in young people of the Surcolombiana University of the subject 'formative sport', semester 2013-2

\section{Abstract}

The research called "Effects of an educational intervention strategy on the development of healthy lifestyles and habits among young people of the Surcolombiana University of the subject of training sport semester B 2013." It is a work carried out by students of the chair of formative sports of Surcolombiana University, to encourage physical activity as a habit so that it can be assimilated into the lifestyle of young university students, some parameters of physical condition were used to perceive favorable changes and, in this way, generate gratification, the intervention also used a methodology with a high ludic component in order to make the class more attractive. The results show the benefits of the intervention when statistically significant changes were found in many of the variables studied.

Keywords: healthy lifestyle, training sport, university students, pathogenic behaviors, immunogenic behaviors. 


\section{Introducción}

Las conductas en el marco de la salud se pueden considerar como adecuadas o inadecuadas. Ellas determinan un estilo de vida de riesgo o por el contrario de protección. Las inadecuadas, denominadas patógenos conductuales -término utilizado por (Piqueras Rodriguez \& Martínez Gonzalez, 2008) - ó conductas patógenas - son todas aquellas conductas que afectan la salud de los individuos aumentando el riesgo de enfermar y causar fallecimiento. Por el contrario, las conductas adecuadas, denominadas inmunógenos conductuales o conductas inmunógenas, son todas aquellas conductas que protegen la salud y disminuyen el riesgo de enfermar.

Muchas de las conductas patógenas no son percibidas como riesgosas por los universitarios, ya que un cuerpo joven puede asimilar algunos excesos propios de esta edad, sin embargo, sí estos hábitos persisten, no adoptará un estilo de vida saludable, y terminará perjudicando su salud para etapas posteriores de su vida. Un obstáculo para provocar un cambio de conducta en los jóvenes, es que no atienden argumentos de tipo utilitario para reaccionar favorablemente, los jóvenes atienden razones de tipo emotivo, porque les gusta, les genera placer, disfrute, alegría etc, no porque determinada actividad la consideren beneficiosa.

Contrarrestar el sedentarismo propio de nuestra sociedad actual es uno de los propósitos de la educación física y de manera específica de la cátedra de deporte formativo que se imparte en todos los programas de la universidad. La actividad física es una dimensión de salud que puede condicionar otras dimensiones, sin embargo, para que esta sea saludable, debe generar gratificación de tipo emocional, que el estudiante la perciba como divertida.

Este fue el propósito de la experiencia investigativa cuyos resultados exponemos en el informe denominado "Efectos de una estrategia de intervención educativa en el desarrollo de hábitos y estilos de vida saludable en jóvenes de la Universidad Surcolombiana de la asignatura deporte formativo semestre B 2013". La propuesta de intervención consideró las unidades temáticas: beneficios de la actividad física, comohábito dentro del estilo de vida saludable y componentes de la aptitud física, todos ellos desarrollados en las clases en un ambiente lúdico. En este sentido los resultados evidenciaron la influencia de la variedad de posibilidades de actividad física sobre las diferentes áreas de la aptitud física como la aptitud cardiorespiratoria, la composición corporal, el fortalecimiento muscular, la flexibilidad y la aptitud neuromotora en la motivación hacia la práctica de la actividad física.

Se adaptaron y utilización principios y elementos propios del entrenamiento deportivo en la clase de deporte formativo con el fin de invitar a reflexionar a los estudiantes para que incorporen la actividad física como una dimensión que contribuye a la adopción de un estilo de vida saludable.

\section{Materiales y métodos}

Dado que la pretensión fue la de especificar las propiedades y caraterísticas de la actividad física de los estudiantes universitarios, se utilizó el método descriptivo del paradigma cuantitativo, este método permitió medir, evaluar y recolectar información sobre las variables estudiadas.

\section{Población}

Los estudiantes matriculados de los diferentes programas de la Universidad Surcolombiana que cursaban la asignatura Deporte Formativo.

\section{Delimitación de la población}

Los estudiantes que cursaron la asignatura de deporte formativo en el segundo semestre de 2013. Ocho cursos de 45 estudiantes cada uno. Es decir 360 estudiantes.

\section{Muestra}

Un grupo de estudio de 30 estudiantes y un grupo de control de 30 estudiantes.

\section{Instrumentos}

Los instrumentos que se utilizaron en la investigación fueron: 
- Contenidos programáticos dela intervención.

- Para evaluar la dinámica de la clase con enfoque lúdico, se aplicó una encuesta considerando aspectos como la metodología, la coherencia, los materiales, las actividades.

- Para valorar los diferentes parámetros de la condición física, se aplicaron una serie de test al iniciar y al finalizar el semestre, para determinar la incidencia de las actividades desarrolladas durante el curso de deporte formativo sobre la condición física del estudiante.

\section{Test del kilómetro (1000 mt.)}

Objetivo: Valorar la resistencia aeróbicaanaeróbica.

Material e instalaciones: Cronometro. Pista de atletismo o terreno llano sin muchas curvas perfectamente delimitado. (Vallodoro, Eric, 2010).

\section{Test de fuerza abdominal}

Objetivo: Determinar la fuerza a la resistencia en la zona abdominal.

Equipo: Cronómetro y entrenador.

Buscar el mayor número de repeticiones en un minuto. (Test y cuestionarios).

\section{Test de fuerza de brazos}

Objetivo: Determinar de forma general la fuerza a la resistencia del pectoral, deltoides y tríceps realizando el mayor número de flexiones de brazos de cubito abdominal en 1 minuto.

\section{Equipo: Cronómetro}

\section{Medidas antropométricas}

Se considera a la antropometría como la ciencia que estudia las medidas del cuerpo humano, con el fin de establecer diferencias entre individuos, grupos, razas, etc. (Sillero, Quintana, 2005)

\section{Peso corporal}

Definición: En sentido estricto, debería usarse el término de masa corporal en lugar de peso corporal.

Instrumental: Báscula o balanza pesa personas. La medida del peso corporal se expresa en kilos (kg.), con una precisión de $0.1 \mathrm{~kg}$.

\section{Estatura}

Definición: La estatura se define como la distancia que existe entre el vértex y el plano de sustentación. También se le denomina como talla en bipedestación o talla de pie, o simplemente como talla.

Instrumental: Estadiómetro. La medida de la estatura se expresa en centímetros $(\mathrm{cm})$, con una precisión de $1 \mathrm{~mm}$.

\section{Indice de Masa Corporal}

En nombre de IMC se usa desde hace relativamente poco tiempo, tanto como desde 1972 cuando Ancel Keys asignó el nombre literal de Body Mass Index (Indice de Masa Corporal) a un patrón matemático que ya se conocía desde hacia mucho tiempo como fórmula de Quetelet.

El índice de masa corporal (IMC) es un indicador simple de la relación entre el peso y la talla que se utiliza frecuentemente para identificar el sobrepeso y la obesidad en los adultos. Se calcula dividiendo el peso de una persona en kilos por el cuadrado de su talla en metros $(\mathrm{kg} / \mathrm{m} 2)$.

Sin lugar a dudas, la principal ventaja es su sencillez para obtener las variables peso y talla, para aproximar la situación ponderal del individuo sin demasiadas complicaciones matemáticas. A dia de hoy, los criterios de la OMS y de la mayoría de sociedades y autoridades sanitarias consideran que se tiene...

\section{Procedimiento}

Se desarrolló el siguiente procedimiento, el cual es acorde a los planteamientos de los objetivos propuestos:

- Se definió la muestra de participantes entre dos grupos de cursos diferentes, en uno se le hizo la intervención (grupo experimental) y en el otro grupo se hicieron las actividades normales (grupo de control).

- Hay que destacar que la intervención se hizo con las actividades propias de la clase de deporte formativo, inculcando la importancia de la actividad física como dimensión de salud y la necesidad de cumplir el compromiso de realizar además de las dos sesiones semanales, otra sesión en el tiempo del grupo experimental.

- Los estudiantes participaron de las actividades generales de la clase, pero el grupo 
experimental además se comprometió a realizar actividades físicas extra-clase a partir de las instrucciones recibidas.

- Capacitación a evaluadores: A los estudiantes participantes que contribuyeron a la recolección de la información se les capacitó en el manejo de la batería a aplicar con sus diferentes pruebas.

- Calibración de evaluadores: se realizarán sesiones prácticas donde se realicen los ajustes necesarios para el proceso de calibración de evaluadores teniendo en cuenta criterios intraevaluador e interevaluador.

- Prueba piloto: se realizó una prueba de ajuste con 15 estudiantes para conocer el rodaje de la prueba.

- Recolección de la información. Previo a la aplicación de la batería se realizó el diligenciamiento del consentimiento informado.

\section{Plan de análisis}

La sistematización de la información se realizó en el programa SPSS versión 23. Para establecer la normalidad dado que son variables cuantitativas se aplicó Shapiro-Wilk y la $t$ student para muestras relacionadas, cuando las muestras provenían de una distribución normal, y de wilcoxon cuando no provenían de una distribución normal.

\section{Resultados}

La tabla No. 1 ilustra la edad de los estudiantes participantes en el grupo experimental.

Tabla 1. Frecuencia y porcentaje de la edad de los estudiantes

\begin{tabular}{c|c|c|c|c|c}
\hline \multicolumn{2}{c}{ Edades } & \multirow{2}{*}{ Frecuencia } & Porcentaje & $\begin{array}{c}\text { Porcentaje } \\
\text { válido }\end{array}$ & $\begin{array}{c}\text { Porcentaje } \\
\text { acumulado }\end{array}$ \\
\hline \multirow{5}{*}{ Válido } & 17 & 5 & 16,7 & 16,7 & 16,7 \\
\cline { 2 - 6 } & 18 & 8 & 26,7 & 26,7 & 43,3 \\
\cline { 2 - 6 } & 19 & 3 & 10,0 & 10,0 & 53,3 \\
\cline { 2 - 6 } & 20 & 5 & 16,7 & 16,7 & 70,0 \\
\cline { 2 - 6 } & 22 & 1 & 10,0 & 10,0 & 80,0 \\
\cline { 2 - 6 } & 23 & 1 & 3,3 & 3,3 & 83,3 \\
\cline { 2 - 6 } & 24 & 2 & 6,7 & 3,3 & 93,3 \\
\cline { 2 - 6 } & 27 & 2 & 6,7 & 6,7 & 100,0 \\
\cline { 2 - 6 } & Total & 30 & 100,0 & 100,0 & \\
\hline
\end{tabular}

La tabla No. 2 se observa el juicio valorativo de los estudiantes sobre la coherencia de la clase.

Tabla 2. Coherencia en el desarrollo de la clase

\begin{tabular}{c|c|c|c|c}
\hline & Frecuencia & Porcentaje & $\begin{array}{l}\text { Porcentaje } \\
\text { válido }\end{array}$ & $\begin{array}{l}\text { Porcentaje } \\
\text { acumulado }\end{array}$ \\
\hline Muy buena & 13 & 43,3 & 43,3 & 43,3 \\
\hline Buena & 13 & 43,3 & 43,3 & 86,7 \\
\hline Algo buena & 4 & 13,3 & 13,3 & 100,0 \\
\hline Total & 30 & 100,0 & 100,0 & \\
\hline
\end{tabular}

La tabla No. 3 expone el grado de satisfacción de los estudiantes sobre el material utilizado en la clase.

Tabla 3. Material utilizado en la clase

\begin{tabular}{c|c|c|c|c|c}
\hline \multicolumn{2}{c|}{} & Frecuencia & Porcentaje & $\begin{array}{c}\text { Porcentaje } \\
\text { válido }\end{array}$ & $\begin{array}{c}\text { Porcentaje } \\
\text { acumulado }\end{array}$ \\
\hline \multirow{3}{*}{ Válido } & Muy bueno & 13 & 43,3 & 43,3 & 43,3 \\
\cline { 2 - 6 } & Bueno & 15 & 50,0 & 50,0 & 93,3 \\
\cline { 2 - 6 } & Algo bueno & 2 & 6,7 & 6,7 & 100,0 \\
\cline { 2 - 6 } & Total & 30 & 100,0 & 100,0 & \\
\hline
\end{tabular}

En la tabla No. 4 se aprecia el nivel de aceptación de las actividades realizadas en la clase.

Tabla 4. Valoración de las actividades realizadas en clase

\begin{tabular}{c|c|c|c|c|c}
\hline Actividades & $\begin{array}{c}\text { Muy } \\
\text { agradable }\end{array}$ & Agradable & $\begin{array}{c}\text { Algo } \\
\text { agradable }\end{array}$ & $\begin{array}{c}\text { Poco } \\
\text { agradable }\end{array}$ & $\begin{array}{c}\text { Nada } \\
\text { Agradable }\end{array}$ \\
\hline Caminata pista USCO & 8 & 12 & 8 & 2 & 0 \\
\hline Caminata urbana & 8 & 15 & 4 & 0 & 0 \\
\hline Carrera continua & 7 & 15 & 7 & 1 & 0 \\
\hline Gimnasio de fuerza & 14 & 15 & 0 & 1 & 0 \\
\hline Gimnasio Bio-Saludable & 15 & 10 & 5 & 0 & 0 \\
\hline $\begin{array}{c}\text { Pesas con botellas de } \\
\text { arena }\end{array}$ & 10 & 10 & 8 & 2 & 0 \\
\hline Elásticos con neumático & 9 & 9 & 10 & 2 & 0 \\
\hline Bastones & 5 & 15 & 7 & 1 & 2 \\
\hline Aeróbicos & 13 & 12 & 2 & 2 & 1 \\
\hline Spinning & 11 & 12 & 5 & 2 & 0 \\
\hline Baile & 16 & 9 & 2 & 3 & 0 \\
\hline Futbol de salón & 11 & 15 & 3 & 1 & 0 \\
\hline Voleibol & 13 & 9 & 5 & 2 & 1 \\
\hline Futbol & 18 & 3 & 6 & 3 & 0 \\
\hline Baloncesto & 9 & 14 & 4 & 2 & 1 \\
\hline Ultímate & 17 & 10 & 3 & 0 & 0 \\
\hline Rugby & 13 & 8 & 3 & 6 & 0 \\
\hline Balón mano & 7 & 15 & 6 & 2 & 0 \\
\hline Juegos populares & 16 & 8 & 6 & 0 & 0 \\
\hline Juegos recreativos & 16 & 9 & 5 & 0 & 0 \\
\hline
\end{tabular}

IMC grupo Experimental

Como se observa en la gráfica hay una diferencia de las medias entre la primera y segunda toma en el grupo experimental (Tabla No. 5). 
Tabla 5. IMC. Muestras relacionadas

\begin{tabular}{|c|c|c|}
\hline \multicolumn{3}{|l|}{ Descriptivos } \\
\hline & & Estadístico \\
\hline IMC EXPERIMENTAL PRIMERO & Media & 22,83 \\
\hline IMC EXPERIMENTAL SEGUNDO & Media & 21,60 \\
\hline
\end{tabular}

Posteriormente se aplicó en el grupo experimental la prueba $\mathrm{T}$ de Student para muestras relacionadas para establecer si la diferencia entre las dos tomas del IMC es estadísticamente significativa. (Tabla 6).

Tabla 6. Prueba de muestras emparejadas

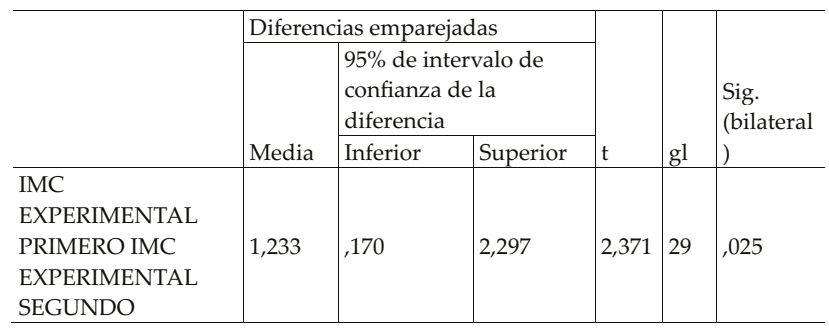

Se estableció que hub una diferencia significativa en las medias de los IMC antes y después de la intervención. Por lo tanto se concluye que la intervención si tuvo efectos significativos sobre el IMC de los estudiantes. Dado que el P-valor o Sig. está por debajo de alfa, 0,025. Como lo demuestra la tabla de muestras emparejadas. De hecho los estudiantes en promedio bajaron 1,23 puntos su IMC.

\section{IMC grupo Control}

Al grupo control se le dio un tratamiento estadístico igual que al grupo experimental en la valoración del IMC en la intervención. (Tabla 7).

Tabla 7. Prueba de normalidad IMC grupo control

\begin{tabular}{l|l|l|l}
\hline & \multicolumn{3}{|l}{ Shapiro-Wilk } \\
\hline & Estadístico & gl & Sig. \\
\hline IMC CONTROL PRIMERO &, 890 & 30 &, 005 \\
\hline $\begin{array}{l}\text { IMC CONTROL } \\
\text { SEGUNDO }\end{array}$ &, 945 & 30 &, 128 \\
\hline
\end{tabular}

Se observa en la gráfica una pequeña diferencia de las medias entre el primera $y$ segunda toma en el grupo control, por lo que se aplica el T student para ver si esta diferencia es significativa. (Tabla 8).
Tabla 8. Media IMC dos momentos

\begin{tabular}{l|l|l}
\hline & Media & $\mathrm{N}$ \\
\hline IMC CONTROL PRIMERO & 22,63 & 30 \\
\hline IMC CONTROL SEGUNDO & 22,17 & 30 \\
\hline
\end{tabular}

En el grupo Control, aunque la diferencia es de centésimas, también se acoge la hipótesis alternativa en el sentido de que hay diferencia estadísticamente significativa ya el $\mathrm{P}$-valor fue de 0,006 , con lo que se concluye que la intervención en este grupo, también tuvo efectos suficientes, para provocar una disminución del IMC en los estudiantes. (Tabla 9).

Tabla 9. Prueba de muestras emparejadas

\begin{tabular}{|c|c|c|c|}
\hline & $\begin{array}{c}\text { Diferencias } \\
\text { emparejadas }\end{array}$ & & Sig. \\
\hline & $\mathrm{t}$ & $\mathrm{gl}$ & (bilateral) \\
\hline $\begin{array}{l}\text { IMC CONTROL PRIMERO - IMC } \\
\text { CONTROL SEGUNDO }\end{array}$ & 2,971 & 29 & ,006 \\
\hline
\end{tabular}

\section{Fuerza abdominal. Grupo Experimental}

La T Student para muestras relacionadas corroboró que hay una diferencia significativa en las repeticiones de abdominales entre la primera y segunda toma del test de abdominales, por lo que se puede concluir que la intervención tuvo efectos positivos sobre la fuerza abdominal de los estudiantes. (Tabla 10).

Tabla 10. Prueba de muestras emparejadas

\begin{tabular}{l|l|l|l|l}
\hline \multicolumn{2}{l|}{} & $\mathrm{t}$ & $\mathrm{gl}$ & $\begin{array}{l}\text { Sig. } \\
\text { (bilateral } \\
\text { ) }\end{array}$ \\
\hline Par 1 & $\begin{array}{l}\text { ABDOMINALES EXP PRIMERO } \\
\text { ABDOMINALES EXP } \\
\text { SEGUNDO }\end{array}$ & $-9,433$ & 29 &, 000 \\
\hline
\end{tabular}

\subsection{0 metros. Resistencia aeróbica- anaeróbica. Grupo experimental}

En el cuadro se aprecia la media de la segunda aplicación fue menor, sin embargo, dado que el valor o la significancia, es menor que el P-valor, se concluye que los datos no provienen de una distribución normal, por lo tanto no se puede aplicar la prueba T-student para muestras relacionadas. (Tabla 11). 
Tabla 11. Prueba de normalidad

\begin{tabular}{l|l|l|l|l|l|l}
\hline & \multicolumn{5}{|l}{ Kolmogorov-Smirnov $^{\mathrm{a}}$} & \multicolumn{3}{l}{ Shapiro-Wilk } \\
\cline { 2 - 7 } & $\begin{array}{l}\text { Estadístic } \\
\mathrm{o}\end{array}$ & $\mathrm{gl}$ & Sig. & $\begin{array}{l}\text { Estadístic } \\
\mathrm{o}\end{array}$ & $\mathrm{gl}$ & Sig. \\
\hline MIL METROS EXP PRIMERO &, 191 & 30 &, 007 &, 927 & 30 &, 042 \\
\hline MIL METROS EXP SEGUNDO &, 168 & 30 &, 030 &, 797 & 30 &, 000 \\
\hline
\end{tabular}

En lugar de medias, se comparan medianas, aplicando la prueba de Wilcoxon. (Tabla 12).

Tabla 12. Estadisticos de prueba

\begin{tabular}{l|l}
\hline & $\begin{array}{l}\text { MIL METROS EXP PRIMERO - } \\
\text { MIL METROS EXP SEGUNDO }\end{array}$ \\
\hline$Z$ & $-3,893^{\mathrm{b}}$ \\
\hline Sig. asintótica (bilateral) &, 000 \\
\hline \multicolumn{2}{l}{ a. Prueba de rangos con signo de Wilcoxon } \\
\hline
\end{tabular}

La prueba de wilcoxon, arrojó como resultado que hubo una diferencia significativa entre las medianas entre la primera y segunda aplicación de la prueba de 1.000 metros para mirar la resistencia aeróbica-anaeróbica, por lo tanto se concluye que la intervención incidió en una mejora de esta capacidad en los estudiantes.

\section{Discusión}

La definición de la OMS, sobre forma física es "la capacidad de una persona para realizar satisfactoriamente el trabajo muscular" (Sapagi $\mathrm{J}, 2007)$. Para un atleta de alta competencia estar en forma es ser capaz de ganar una prueba, mientras que para un anciano estar en forma es ser capaz de caminar o valerse por sí mismo.

La actividad física y el ejercicio físico se han convertido hoy en día en elementos primordiales de prevención de enfermedades, de tal forma que hoy ayudan a combatir las secuelas o la pobre calidad de vida que deja tras de sí algunas enfermedades que ha sufrido la persona y en este orden de ideas no hay que olvidar que el ejercicio físico es un entrenamiento al que se somete el organismo para que funcione cada vez mejor (Bauman AE, 2012).

Por todas las anteriores consideraciones la actividad física es objeto de estudio en investigaciones e intervenciones de la comunidad científica, la intervención pudo constatar las bondades de la actividad física al provocar un cambio en las variables estudiadas, en el grupo de estudiantes, esta intervención tenía una doble connotación, desde la salud y desde la educación, normalmente asumimos que con el hecho de hacer actividad física, estamos "per se" contribuyendo a la salud, ignorando otros componentes como el educativo para ir creando un valor agregado a nuestra misión e ir subrayando su importancia.

En este sentido la investigación denominada Comportamiento y salud de los jóvenes universitarios: satisfacción con el estilo de vida (Lema Soto Luisa Fernanda, 2009) con relación a la actividad física sus resultados no difieren mucho de estudios similares, Las puntuaciones obtenidas indican que los universitarios llevan a cabo prácticas saludables en la mayoría de las dimensiones de los estilos de vida, con excepción la de ejercicio y actividad física, ya que el promedio de la calificación es estadísticamente diferente del punto medio de la dimensión, con un nivel de significación del $5 \%$. Los principales comportamientos que se destacan como menos saludables en esta área son: la poca o nula realización de algún ejercicio o práctica corporal durante al menos 30 minutos, tres veces a la semana $(77 \%)$, y caminar o montar bicicleta en lugar de tomar otro medio de transporte si necesita movilizarse a un lugar cercano $(67,5 \%)$. Según el sexo, hubo diferencias significativas, teniendo prácticas más saludables los hombres que las mujeres $(Z=-6,184 ; p=0,000$; prueba Mann-Whitney). En cuanto al nivel de satisfacción con esta dimensión, el $(51,4) \%$ afirma que se siente satisfecho con sus prácticas de ejercicio y actividad física, el $(35,9 \%)$ se siente medianamente satisfecho y el 11,9\% nada satisfecho. A medida que aumenta el nivel de satisfacción, los estudiantes realizan prácticas de ejercicio y actividad física más saludables ( $\mathrm{x} 2=$ 44,889; $\mathrm{p}=$ 0,000; prueba Kruskal-Wallis).

En la investigación Índice de masa corporal, frecuencia y motivos de estudiantes universitarios para realizar actividad física, (Mollinedo Montaño Fabiana Esther, 2013) Solo el $41,2 \%$ de los estudiantes practicaba en uno o varios deportes, $91,2 \%$ más de media 
hora al día, aunque de estos solo el 30,5\% lo realizaba al menos 5 días a la semana. El deporte más practicado fue el fútbol soccer $(13,3 \%)$, seguido de levantamiento de pesas $(10,3 \%)$ y cardiofitness (10,3\%); en su mayoría practican deporte en lugares públicos $(33,8 \%)$, en un gimnasio $(31,8 \%)$ o en su casa $(23,5 \%)$; el $37,7 \%$ lo practica en grupo, seguido de un $33,2 \%$ que lo hacía solo. Los principales motivos para realizar ejercicio fueron por salud $(50,8 \%)$, por diversión y pasar el tiempo $(29,1 \%)$, por gusto $(26,2 \%)$ y estética $(17,9 \%)$.

Por otro lado al indagar las actividades realizadas durante el tiempo libre el 81,7\% respondió ver televisión, 86,1 \% estar en la computadora, $77 \%$ jugar videojuegos, $83,1 \%$ estar con los amigos, 88,4\% convivir con la familia. Entre los que no realizaban ejercicio físico, las principales razones fueron por no tener tiempo $(49,7 \%)$, porque $\mathrm{n}$ hay instalaciones deportivas cerca $(29,5 \%)$ o porque terminan muy cansados de la escuela $(20,9 \%)$.

De la actividad física entre otras cosas se espera que: "contribuya al mejoramiento de la condición de salud, aumente la capacidad productiva, mejorelas relaciones interpersonales, brinde alternativas sanas para el uso del tiempo libre, compense el sedentarismo de la actividad laboral y de orden intelectual de los sujetos" (Hernández A, 2010). Para cumplir con lo mencionado es necesario:

- Adecuar las capacidades e intereses del individuo (individualización)

- Practicar con regularidad mínimo tres veces por semana (continuidad).

- Generar una adaptación biológica sin producirdaño(adecuación deestímulo, aumento gradual de la carga).

- Graduar los esfuerzos de manera que aumenten el nivel de rendimiento (ondulación).

Estamos frente a un rico objeto de estudio que basa su pertinencia en la urgente necesidad de promover la actividad física frente al estilo de vida sedentario propio de nuestra sociedad actual, además de otras consideraciones de tipo científico e investigativo en la cual se pueden poner al servicio de la población en general, los aportes dados por el entrenamiento deportivo en sus estudios que pretenden incrementar la condicion física y el rendimiento humano.

\section{Conclusiones}

- Se determinó que hubo una mejora estadisticamente significativa de las medias de los IMC antes y después de la intervención. Por lo tanto se concluye que la intervención si tuvo efectos significativos sobre el IMC de los estudiantes que participaron en el estudio. Lo cual ratifica la hipotesis alternativa, dado que el P-valor o Sig. esta por debajo de alfa, 0,025. De esta manera los estudiantes en promedio bajaron 1,23 puntos su IMC.

- El logro anterior mejoró la condición física de los estudiantes, la resistencia aeróbica, fuerza de brazos y abdominal, además se inculcó la importancia de la actividad física como dimensión muy importante en un estilo de vida saludable.

- La actividad física como dimensión de salud se vio fortalecida con la intervención como lo evidencia los resultados de las valoraciones cuantitativas realizadas, y la percepción observada en la encuesta.

- Como se aclaró en el diseño metodológico, la intervención respondía a la lógica de unas clases con fines educativos y al compromiso de realizar actividad física en otra sesión semanal por parte de los estudiantes del grupo experimental, no a la metodología de un entrenamiento deportivo que pretende el rendimiento, por lo tanto no se esperaban grandes modificaciones en las variables evaluadas.

- Concluida la escolaridad media, el estudiante hace muy poca actividad física o sin la frecuencia e intensidad requerida dada su edad, es decir, el estudiante no tiene un hábito arraigado para continuar de manera voluntaria haciendo actividad física o deporte.

- Uno de los logros del programa fue cambiar la concepción traída desde la secundaria, en el cual se veía al deporte como único medio para realizar actividad física dejando de lado todos los aportes que a traves de diferentes actividades físicas. Al respecto se dio un amplio repertorio de posibilidades tanto individuales 
como colectivas con un alto contenido lúdico para hacer actividad física.

- Se espera que la clase de deporte formativo trascienda el activismo, y aporten conceptos teóricos con criterio científico para que se pueda dar la auto prescripción de actividad física por parte del estudiante.

- Se considera que se pueden romper los esquemas y ambientes de las demás áreas al realizar la actividad física de manera gradual y progresiva utilizando las actividades físicas como una gratificación y no un castigo.

- Los test de Condición Física demostraron que las actividades realizadas en el curso tuvieron incidencia sobre los estudiantes y evidenciaron una atención especial a la condición física, además, en algunos se observó una mejoría en su aptitud física.

- Las actividades y las encuestas propiciaron reflexiones sobre hábitos no solo de actividad física, sino en otras dimensiones como alimentación, consumo de alcohol, tabaco y otras drogas, y auto concepto.

- El hecho de que una persona reconozca como adecuada la práctica de la actividad física para la salud no constituye un factor decisivo para que realice la misma. La sola percepción individual no determina el comportamiento, para incentivar la actividad física en los estudiantes, dada su juventud, no hay que recurrir a razones utilitarias, como es buena para la salud y la vitalidad, sino a través de sus emociones, la alegría, el goce, el disfrute, la diversión y el placer que estas actividades generan en compañía de amigos.

- Hay que considerar la influencia que puede tener la realización de actividad física en el resto de las dimensiones de la salud y en la apropiación de mejores hábitos de vida en el estudiante, para poder considerar a la misma como promotora de estilo de vida saludable no solo por sus aportaciones directas, como se ha indicado antes, sino por eliminar hábitos insaludables y potenciar hábitos saludables. El practicante regular de actividad física generalmente cuida de su alimentación, se abstiene de consumir alcohol, tabaco u otras drogas, y en general crea un entorno amigable y saludable que promueve y protege su salud.
- Sin embargo el tiempo de la intervención y el enfoque dado a la dimensión de la actividad física, no son suficientes para generar un cambio de conducta como para cambiar hábitos ya arraigados en los estudiantes como el consumo de alcohol, tabaco $u$ otras drogas. Por otra parte se debe resaltar que aunque no es suficiente, se comienza a dar una reflexión en torno a esos temas, para gestar algún cambio en el estilo de vida.

\section{Referencias Bibliográficas}

Bauman AE, R. R. (2012). Correlates of physical activity: why are some people physically active and others not? Lancet. 2012; 3. Lancet, 80 (13), 258-271.

Hernández A, G. L. (2010). Ambientes urbanos y actividad física en adultos mayores: Relevancia del tema para América Latina. Rev. salud pública, 12 (2), 327-335.

JC., E. (2002). Promoción y Prevención en el Sistema de Salud en Colombia. Rev. Salud pública, 12 (3), 1 -12.

Lema Soto Luisa Fernanda, S. T. (2009). Comportamiento y salud de los jóvenes universitarios: satisfacción con el estilo de vida. (P. U. Javeriana., Ed.) Pensamiento Psicológico, 5 (12), 71-88.

Mollinedo Montaño Fabiana Esther, T. O. (2013). Índice de masa corporal, frecuencia $y$ motivos de estudiantes universitarios para realizar actividad física. Educación Médica Superior, 27 (3).

Piqueras Rodríguez, J. A., \& Martínez González, A. E. (2008). Ansiedad Depresión y Salud. Suma Psicológica, Vol. 15 N$^{\circ} 1$ Marzo de 2008, 43-74, ISSN 0121-4381, 15 (1), 43-74.

Sapagi J, K. I. (2007). Capital social y promoción de la salud en América Latina. Rev. Saúde Pública, 41 (1), 139-49.

Sillero, Quintana. (6 de 2005). Facultad de Ciencias de la actividad física y del deporte. 
(INEF) Universidad Politécnica de Madrid. Recuperado el 8 de Noviembre de 2014, de http://ocw.upm.es/educacion-fisica-ydeportiva/kinantropometria/contenidos/ temas/Tema-2.pdf

Test y cuestionarios. (s.f.). http://testsworld.net/. Recuperado el 12 de Noviembre de 2014, de http://es.testsworld.net/tag/test-defuerza-abdominales

Vallodoro, Eric. (3 de Noviembre de 2010). Entrenamiento deportivo.worldpress.com. Recuperado el 7 de Octubre de 2014, de https://entrenamientodeportivo. wordpress.com 